\title{
A Deep Search for Planets in the Inner 15 au around Vega
}

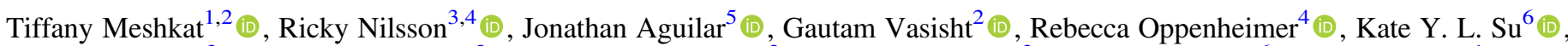

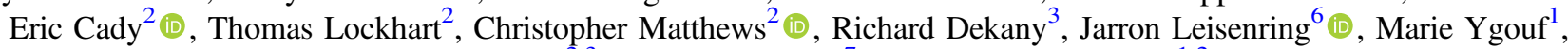 \\ Dimitri Mawet $^{2,3}$ (D) Laurent Pueyo ${ }^{7}$, and Charles Beichman ${ }^{1,2}$ \\ ${ }^{1}$ IPAC, California Institute of Technology, M/C 100-22, 1200 East California Boulevard, Pasadena, CA 91125, USA \\ 2 Jet Propulsion Laboratory, California Institute of Technology, 4800 Oak Grove Drive, Pasadena, CA 91109, USA \\ ${ }^{3}$ Department of Astronomy, California Institute of Technology, 1200 East California Boulevard, MC 249-17, Pasadena, CA 91125, USA \\ Astrophysics Department, American Museum of Natural History, Central Park West at 79th Street, New York, NY 10024, USA \\ ${ }^{5}$ Department of Physics and Astronomy, The Johns Hopkins University, Baltimore, MD 21218, USA \\ ${ }^{6}$ Steward Observatory, University of Arizona, 933 N Cherry Avenue, Tucson, AZ 85721, USA \\ Space Telescope Science Institute, 3700 San Martin Drive, Baltimore, MD 21218, USA \\ Received 2018 June 11; revised 2018 September 5; accepted 2018 September 11; published 2018 October 18
}

\begin{abstract}
We present the results of a deep high-contrast imaging search for planets around Vega. Vega is an ideal target for high-contrast imaging because it is bright, nearby, and young with a face-on two-belt debris disk that may be shaped by unseen planets. We obtained $J$ - and $H$-band data on Vega with the coronagraphic integral-field spectrograph Project 1640 (P1640) at Palomar Observatory. Two nights of data were obtained in 2016, in poor seeing conditions, and two additional nights in more favorable conditions in 2017. In total, we obtained 5.5 hours of integration time on Vega in moderate to good seeing conditions $(<1$ !!5). We did not detect any low-mass companions in this system. Our data present the most sensitive contrast limits around Vega at very small separations (2-15 au) thus far, allowing us to place new constraints on the companions that may be sculpting the Vega system. In addition to new constraints, as the deepest data obtained with P1640, these observations form the final legacy of the now decommissioned instrument.
\end{abstract}

Key words: circumstellar matter - planets and satellites: detection - stars: individual (Vega) - techniques: high angular resolution

\section{Introduction}

Nearly all directly imaged planets have been found around stars with bright circumstellar debris disks. These dusty disks contain grains down to a few microns in size, generated in collisional cascades of asteroids and comets (Wyatt 2008) bodies that are the remnants of planetesimals thought to be the building blocks of planet cores. This direct connection between debris disks and planets is seen in several of the currently known planetary systems (HR 8799; Marois et al. 2008, 2010, $\beta$ Pic; Lagrange et al. 2009, 2010, HD 95086; Rameau et al. 2013, 51 Eridani; Macintosh et al. 2015), suggesting that debris disks may be signposts of exoplanetary systems (Raymond et al. 2011, 2012). Meshkat et al. (2017) perform a metaanalysis of new high-contrast imaging data supplemented by archival sensitivity limits to compare the occurrence rate of giant planets in dusty systems versus a well-defined control sample without dust belts under current detection limits. The occurrence of young giant planets around stars with debris disks is shown to be higher than those without debris disks at the $88 \%$ confidence level, suggesting that these distributions are statistically distinct.

An additional hint for the presence of planets may be the signature of two temperatures in the debris disk's spectral energy distribution (SED): a warm inner belt and a cool outer belt. The dust-free gap between these belts may be caused by one or more planets accreting and rejecting the material as they form (Quillen 2006; Chiang et al. 2009). Notably, the HR 8799 planets, $\beta$ Pic b, HD 95086 b, and 51 Eridani b are in two-belt debris disk systems.

Vega is one of the most well-studied stars in the Northern Hemisphere and an ideal target for high-contrast imaging searches. It is a relatively young $(445 \pm 13$ Myr; Yoon et al. 2010) $2.5 M_{\odot}$ A0V star, located $7.68 \pm 0.02 \mathrm{pc}$ away (van Leeuwen 2007). Vega is a bright (0th magnitude) star, making it favorable for good adaptive optics (AO) correction (Metchev et al. 2003) and thus deep contrast limits needed to detect the lowest-mass planets.

Vega has a vast, nearly face-on disk composed of small grains in the form of a disk halo first revealed by Spitzer observations (Su et al. 2005). JCMT 450 and $850 \mu \mathrm{m}$ images (Holland et al. 2017) reveal the smooth, axi-symmetric disk with a deconvolved fitted radius of the disk at 73 and $135 \mathrm{au}$, respectively. These data also suggest that the center of the cold debris belt is offset from the star position by $2^{\prime \prime}$. This offset is smaller than previously detected in interferometric data at $1.3 \mathrm{~mm}$ showing a peak offset of $8^{\prime \prime}-14^{\prime \prime}$ (Koerner et al. 2001; Wilner et al. 2002). Herschel data from 70 to $500 \mu \mathrm{m}$ are consistent with a smooth disk without the peak offset from the star (Sibthorpe et al. 2010). Herschel observations and reanalysis of the Spitzer data reveal emission from an additional component of warm dust near the water ice line ( $\sim 14 \mathrm{au})$, spatially separated from the outer $(\sim 80 \mathrm{au})$ cold belt $(\mathrm{Su}$ et al. 2013). Based on the large gap between the inner warm and outer cold debris, and a companion mass limit from highcontrast imaging searches, Su et al. (2013) suggest that the debris structures in Vega and its twin Fomalhaut system are signposts indicating multiple planets beyond the ice line.

The face-on orientation of the Vega debris disk also makes it an optimal target for exoplanet imaging as a coplanar planet on a circular orbit will always be at the same angular separation from the star. In contrast, the face-on and fast rotating star makes radial velocity searches for planets extremely challenging. Janson et al. (2015) combined deep Spitzer observations 
Table 1

Observing log for Vega Palomar/P1640 data

\begin{tabular}{lccc}
\hline \hline $\begin{array}{l}\text { Observation Dates } \\
\text { UT }\end{array}$ & $\begin{array}{c}\text { Number of } \\
\text { cubes }\end{array}$ & $\begin{array}{c}\text { Integration Time } \\
\text { per ramp (s) }\end{array}$ & $\begin{array}{c}\text { Total Integration } \\
\text { Time (minute) }\end{array}$ \\
\hline 2016 Aug 19 & 137 & 93.0 & 212.4 \\
2016 Aug 20 & 57 & 93.0 & 88.4 \\
2017 Jun 04 & 137 & 93.0 & 212.4 \\
2017 Jun 05 & 115 & 93.0 & 178.3 \\
\hline
\end{tabular}

with MMT observations (Heinze et al. 2008) to constrain planet masses in the Vega system, with an upper limit of $\sim 1-3 M_{\text {Jup }}$ from 100 to 200 au from Spitzer and $\sim 5-20 M_{\text {Jup }}$ from 20 to $80 \mathrm{au}$ from MMT. The limits of $\sim 20 M_{\text {Jup }}$ at 20 au from Heinze et al. (2008) represents the lowest-mass, innermost limits on Vega in the literature. Macintosh et al. (2003) present widefield ( $>50 \mathrm{au}$ ) Keck/NIRC2 $K$-band data with limits down to $\sim 5$ Jupiter masses. Additionally, there is archival Keck/NIRC2 high-contrast imaging data on Vega using a coronagraph with a modest inner working angle of $1^{\prime \prime}$, thus not probing the very inner region of Vega, which remain largely unexplored. As part of the Lyot Project, Hinkley et al. (2007) present observations of the inner regions around Vega, on similar spatial scales to those reachable by $\mathrm{P} 1640$, achieving $H$-band contrasts with mass limits corresponding to 135 and $43 M_{\text {Jup }}$ at 0 ". 5 and $1 . " 0$, respectively.

In this work, we present the results of four nights of Project 1640 (P1640) high-contrast imaging data in the $J+H$ bands. In Section 2, we discuss the observations and data reduction. In Section 3, we show the results of our data reductions and also discuss the detection limits in the context of the Vega debris disk system and compare these limits with previous results.

\section{Observations and Data Reduction}

Vega observations were performed with the P1640 instrument at Palomar Observatory's $5.1 \mathrm{~m}$ Hale telescope over two nights in 2016 (Run 3201, PI: Meshkat) and two nights in 2017 (Run 3372, PI: Meshkat). P1640 (Soummer et al. 2009; Hinkley 2009; Hinkley et al. 2011; Oppenheimer et al. 2012) is a coronagraphic integral-field spectrograph (IFS) with an internal wavefront sensing system (CAL; Zhai et al. 2012; Cady et al. 2013; Vasisht et al. 2014), used in conjunction with the PALM-3000 (P3k; Dekany et al. 2013) extreme AO system at the Hale telescope. The instrument covers a wavelength range of 969-1797 nm, encompassing the near-IR $Y, J$, and $H$ bands in 32 spectral channels, at a spectral resolution of $\Delta \lambda=26.7 \mathrm{~nm}$, with a total field-of-view (FOV) of $\sim 3$ "! $8 \times 3$ " 8 .

As the Hale telescope sits on an equatorial mount, there is no field rotation that can be used for angular differential imaging (ADI; see, e.g., Marois et al. 2006), as is common among other high-contrast imaging instruments. Instead, the IFS allows the wavelength-dependent spatial distribution of speckle noise to be used for spectral differential imaging (SDI; Sparks \& Ford 2002), which can separate real astrophysical sources from the quasi-static speckles. Astrometric satellite spots, introduced by applying a fixed sinusoidal pattern on P3k's deformable mirror (DM), are used for determining the location of the star behind the occulting coronagraphic mask to sub-pixel precision, as well as for photometric calibration. Table 1 lists details about the observations for all four nights, including observation date, number of exposures, integration time per exposure, and total integration time.

\subsection{Data Reduction}

Raw IFS images were processed with the P1640 preprocessing pipeline PCXP (Zimmerman et al. 2011) to extract the 40000 tightly packed spectra and produce data cubes $(x \times y \times \lambda)$. Calibration laser exposures at $1310 \mathrm{~nm}$ and $1550 \mathrm{~nm}$ are combined with sky flats to create a focal plane solution that maps individual spectra to IFS lenslets and corresponding spaxel positions.

We generated post-processed data with two software packages: pyKLIP (Wang et al. 2018) and S4 (Fergus et al. 2014). Both packages perform image registration using the astrometric satellite spots that track the location of the stellar PSF, which is centered behind the focal plane mask. pyKLIP was developed as an instrument-agnostic framework for processing $\mathrm{ADI} / \mathrm{SDI} / \mathrm{ADI}+\mathrm{SDI}$ data with the KLIP PSF subtraction algorithm (Amara \& Quanz 2012; Soummer et al. 2012), and has a P1640 instrument module for processing P1640 data. S4 was developed specifically for processing of P1640 data. The two packages are similar in that they use Principal Component Analysis (PCA) to capture the observed data variance and build a lower dimensional model of the data. However, while pyKLIP uses radially scaled images (where speckles remain stationary in radius-wavelength space) to model the spatial structure of the speckles, S4 models the joint spatial-spectral structure of the non-scaled data, conserving information about the morphology of the quasi-static speckle pattern in each channel. The derived model is subtracted from the data cubes, and the residual data can be inspected (by eye or with more sophisticated PSF matching techniques) to reveal stationary point sources. Each night was processed separately. No significant point sources were detected in the four separate nights of data.

We determined the detection limits achieved in these postprocessed data using three different techniques for contrast estimation, in order to verify the derived accuracy of each method. The first is a detection limit pipeline developed for photometry known as optimized principal component analysis (oPCA; Meshkat et al. 2014), which was adapted to IFS data using the pyKLIP post-processing package. The second is the pyKLIP detection limit pipeline, and the third is a full-field-ofview contrast estimator for S4 residual cubes. The first two pipelines utilize the satellite spots as photometric reference PSFs for fake companion injection. The average of the four satellite spots in each frame is used to create a fake companion, which is injected in the frames before post-processing. The flux and position of the fake companion is scaled in order to determine the $5 \sigma$ detection limit of the data in annuli at different angular separations. By injecting fake companions, we account for self-subtraction as a result of the postprocessing PSF subtraction algorithms. The parameters used in the KLIP analysis were five modes, five annuli, and three subsections. In the following discussion, we present the results from the oPCA pipeline, but we confirmed that these three techniques for determining contrast limits all yield consistent results.

Data for all four nights were processed separately, with a seeing limit cut-off of 1 !' 5 that resulted in $37,33,122$, and 21 cubes from each night, respectively. The resulting signal-tonoise of a fake injected companion is measured in the average 


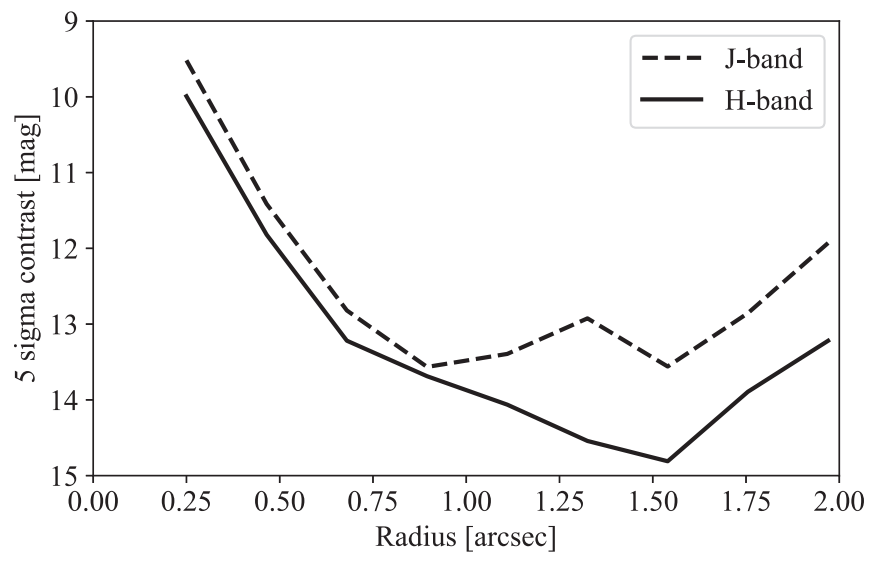

Figure 1. Contrast limits in magnitudes for $J$ (dashed line) and $H$ (solid line) band binned data.

of the four post-processed cubes. In order to maximize the signal from a point source, we binned the IFS data into two "bands", roughly corresponding to $J$ - and $H$-bands.

Figure 1 shows the contrast limits achieved on the average of the four nights using the oPCA pipeline for $J$ - and $H$-bands, where contrast is the flux ratio between the star and a detectable point source.

\subsection{Deep Observation Sensitivity Gain}

Our Vega observations were designed to investigate what imaging contrasts the instrument could deliver in a prolonged sequence ( $\sim 10 \mathrm{hr}$ total exposure) on a bright star; note that the total exposure time over four nights was $11.5 \mathrm{hr}$, out of which only $5.5 \mathrm{hr}$ was deemed usable given the selection criterion and the marginal seeing conditions. The P3k AO system delivers its best possible natural guide star performance on Vega, as the star allows both fast temporal sampling $(1 \mathrm{kHz})$ and high spatial sampling $(64 \times 64$ across the pupil $)$ with negligible photon noise contribution to wavefront estimation (Dekany et al. 2013). In median seeing conditions we expect a post correction wavefront of $\sim 130 \mathrm{~nm} \mathrm{rms}$, corresponding to $J$ - and $H$-band Strehl ratios of 0.65 and 0.78 , respectively; the residual wavefront error is primarily dominated by the AO loop lag.

In the best case scenario, residual noise in post-processed images would be uncorrelated between images in an $n$-image sequence, except in the immediate vicinity of the star at $\leqslant 0$ ". 5 or within $\sim 3.8$ au, where SDI is less effective. In the $H$-band, where the system is optimized, co-adding $n$ images should then provide a near $n^{-1 / 2}$ improvement in detection gains; this translates to a factor of $\sim 14.6$ or 2.9 mag sensitivity over that in a single image. In order to assess the actual gains achieved from co-adding, we create contrast curves for subsets of the data, from $3 \%$ of the data to $100 \%$ (only including data $<1$ "! 5 threshold seeing). Figure 2 shows the contrasts achieved for $3.1 \%, 6.3 \%, 12.5 \%, 25 \%, 50 \%$ and $100 \%$ of the data in $J$ (blue) and $H$-band (red). The solid line indicates the curve with $100 \%$ of the data included, and dashed lines are the subsets of data, with the faintest dashed line representing $3 \%$ of the data. We measure the radial average of the gain in contrast with increasing subset sizes, shown in Figure 3. Our improvement from co-adding frames approximates a $n^{-1 / 5}$ power-law for both $J$ - and $H$-band, shown as dashed lines.

Visual inspection of the post-processed images shows considerable residual structure surviving the standard filtering

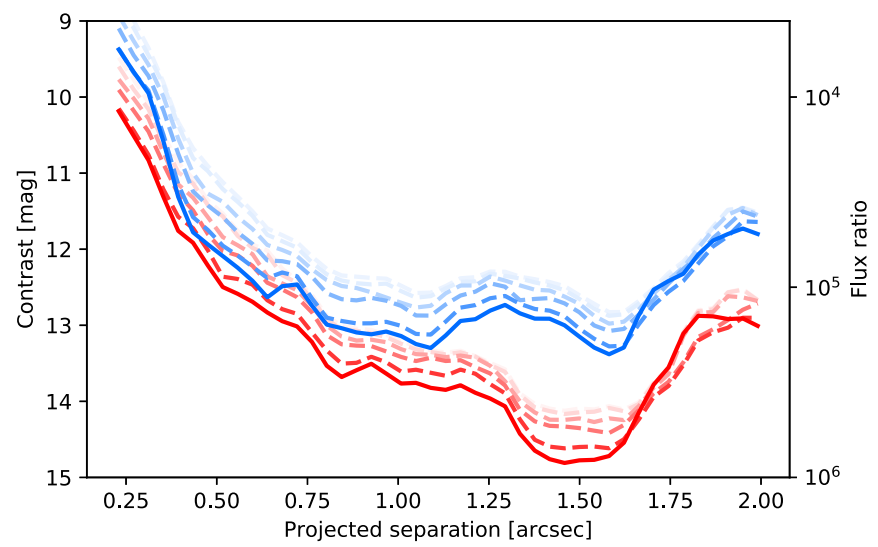

Figure 2. Contrast curves for $J$ - (blue) and $H$-band (red) for $100 \%$ of the data (solid line) and 50\%, 25\%, 12.5\%, 6.3\%, 3.1\% in dashed faded lines. The $3.1 \%$ dashed curve is the faintest curve. Contrast curves generated from subsets of data were computed with a moving average of data.

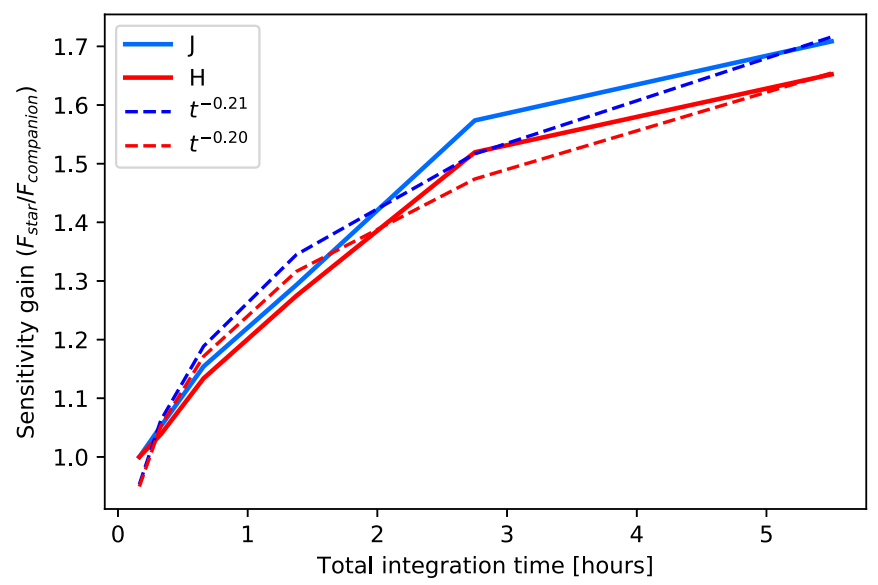

Figure 3. Flux gain factor for $J$ - (blue) and $H$-band (red) over the $5.5 \mathrm{hr}$ integration time. The flux gain is the average over all separations from Figure 2. The dashed lines are best-fit power laws.

and PCA analysis. One clear source of noise is the low lying residual striping pattern due to the H2RG detector arising from temporal gain variations between readout channels. Another source is a band of diffracted light ascribed to a mosaic of under-responsive actuators on the high order deformable mirror (HODM). The changing influence function of these actuators, introduced by aging of the HODM, cause temporal and spatial effects on the contrast that are ill-understood. These structures clearly do not filter or average well, and degrade the azimuthal contrast and its averaging statistics.

A similar deviation from the naively expected $n^{-1 / 2}$ contrast improvement with exposure time in high-contrast AO coronagraphic imaging was demonstrated for the Lyot Project (Hinkley et al. 2007), albeit for smaller dynamic range. This is largely an effect of the Rician distribution of the speckle noise, which retains its non-Gaussian nature after imperfect speckle removal, but will get closer to $n^{-1 / 2}$ as processing techniques like those used by pyKLIP and S4 improve.

\section{Results and Discussion}

\subsection{Companion Limits}

We convert our contrast limits to mass limits using the COND-AMES evolutionary model (Chabrier et al. 2000; 


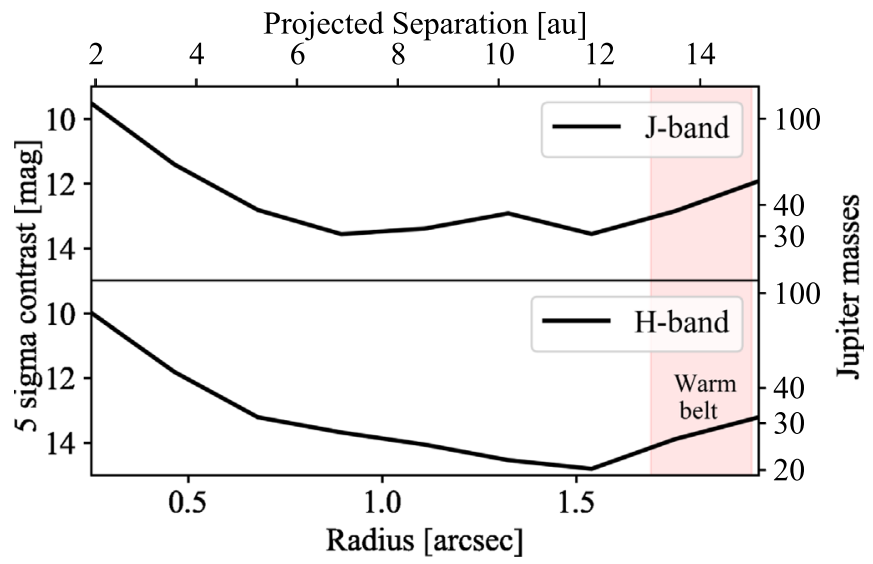

Figure 4. Mass limit plots for the binned $J$ (top) and $H$ (bottom) data. Masses are derived using the COND evolutionary model (Chabrier et al. 2000; Baraffe et al. 2003). The pink region from 13 to 15 au is the approximate location of the warm inner debris belt in the Vega system (Su et al. 2013).

Baraffe et al. 2003). The COND model is used for direct comparison with previous Vega analyses (i.e., Janson et al. 2015). We note that the COND model is limited in that it presents an extreme where the dust opacity has been neglected, simulating a case where there is minimum dust content in the photosphere. At the magnitudes probed by our study, corresponding to effective temperatures of $\sim 2000-1000 \mathrm{~K}$, this may not be completely appropriate, but we have adopted it as a conservative assumption that is consistent with previous studies. Using DUSTY models would change the minimum detectable mass to $\sim 10 M_{\text {Jup. }}$. Figure 4 shows our mass detection limits in $J$ - and $H$-bands, with the approximate location of the warm, inner debris belt marked as a pink region from approximately 13-15 au, inferred from SED fitting ( $\mathrm{Su}$ et al. 2013). These limits allow us to rule out companions responsible for sculpting the inside of the warm debris belt at $\sim 12$ au down to $20 M_{\text {Jup }}$ for $H$-band and $30 M_{\text {Jup }}$ for $J$-band.

These data put limits on the low-mass stellar and brown dwarf companions, which could be responsible for sculpting the Vega debris disks. We compare our results with previous contrast limits from Heinze et al. (2008) with MMT and Janson et al. (2015) with Spitzer in Figure 5. The combined results of these three data sets represents a complete limit of companions from 2 to 200 au. The approximate locations of the warm inner and cold outer disk are labeled in red and blue, respectively.

Figure 6 shows the inner and outer chaotic zones interior to the warm inner belt, assuming that it has an inner boundary at $14 \mathrm{au}$. We adopt the chaotic zone formula, numerically derived by Morrison \& Malhotra (2015) designed for planets at circular orbits assuming high values of planet-to-star mass ratio $(\mu)$. The solid blue line shows the semimajor axis with respect to planet mass for an outer chaotic zone that reaches the inner boundary of the inner belt at 14 au, i.e., the likely location of a circular shepherding planet for a given mass to maintain the inner edge of the warm belt.

Raymond \& Bonsor (2014) perform dynamical simulations to constrain the masses of planets interior to the outer cold debris belt by analyzing planetesimal scattering. This analysis finds that low-mass planets $\left(<1 M_{\text {Jup }}\right)$ orbiting at 5-10 au can be responsible for replenishing the hot exosodiacal dust of the inner belt, suggesting our data are not sensitive enough to place meaningful limits on these simulations.

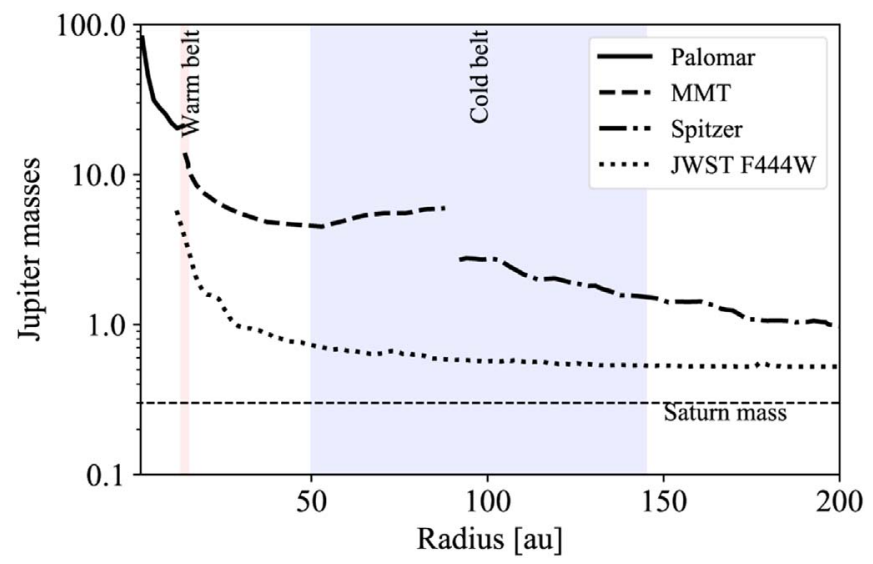

Figure 5. Mass limits around Vega from 2 to $200 \mathrm{au}$, including the binned $H$-band Palomar/P1640 results presented in this work, as well as MMT (Heinze et al. 2008), Spitzer (Janson et al. 2015), and predicted JWST data. All mass limit curves were estimated using the COND-AMES evolutionary model (Chabrier et al. 2000; Baraffe et al. 2003). The debris disk inner and outer belt approximate locations are highlighted in the red and blue regions. The JWST curve reaches a lower mass floor at masses of $0.5 M_{\text {Jup }}$. This mass limit is not physical but due to the mass limit of the COND-AMES model.

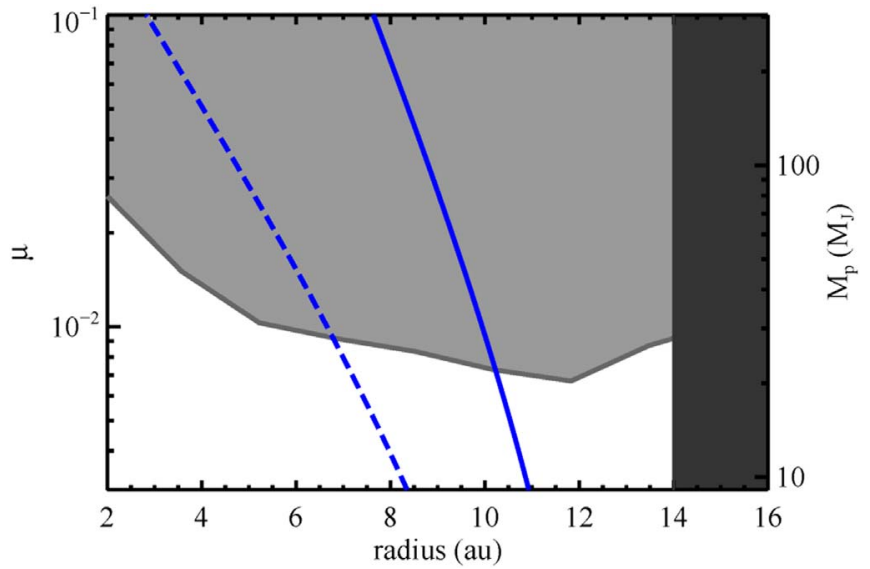

Figure 6. The mass and location of possible circular, shepherding planets (shown as blue solid line) to maintain the inner edge (assumed at $14 \mathrm{au}$ ) of the ice-line belt. The dark gray area marks the location of the inner belt, and the light gray area is the mass regime ruled out by our observation. The blue dashed line is the inner extension of the planet chaotic zone, detailed in Section 3.

\subsection{Debris Disk Limits}

We do not detect the Vega inner debris belt in our data, which is expected to be within our field of view based on SED modeling. Detection of the inner disk is particularly challenging because it is face-on. Absil et al. (2006) detect a $1.29 \pm 0.19 \%$ infrared excess relative to the Vega photosphere with FLUOR/CHARA in $K$-band. If the infrared excess is due to dust grains close to Vega $(<10 \mathrm{au})$, the grains must be very small $(<0.4 \mu \mathrm{m})$, have a fast blow-out time from radiation pressure, and thus requiring a very high replenishment rate. Defrère et al. (2011) confirm this detection with IOTA/IONIC data in $H$-band and suggest this may imply a late heavy bombardment-like event is occurring in the inner region around Vega. We convert our contrast limits to surface brightness limits, in order to determine if we can set limits on the amount of grains being blown out by radiation pressure. Adopting the total mass required for the $H$-band excess from Defrère et al. (2011) and assuming $0.2 \mu \mathrm{m}$ silicate-like grains (albedo of 0.7 at $H$-band), the expected scattered light from these 
blow-out grains at 0 " 5 from the star is more than 2 orders of magnitude fainter than our contrast limit. Detecting the blowout small grains in scattered light will be extremely challenging.

\subsection{Future Observations}

The planned JWST NIRCam GTO observations of Vega (PI Beichman, see Beichman et al. 2010) will provide very deep coronagraphic data searching for companions beyond 1"!5. Figure 5 demonstrates the deep sensitivity limits, which are predicted to be achieved with the JWST NIRCam F444W filter. These data were generated using the python ETC and simulator for JWST NIRCam (pyNRC ${ }^{8}$ ), which uses PSFs derived from WebbPSF (Perrin et al. 2014) paired with NIRCam's instrument performance to simulate a planned observation. The data presented here use the planned observation sequence for the GTO program with an exposure time of $1800 \mathrm{~s}$, two roll positions, and assume a conservative wavefront error drift of $10 \mathrm{~nm}$ between Vega and its linked reference target. Only simple reference subtraction was performed to produce the NIRCam contrast curves. More advanced post-processing techniques, such as PCA, should further improve detection limits interior to Vega's cold belt. The contrast curve is truncated shortward of 1". 5 because Vega will saturate this inner region before the first read. We convert the contrast to masses using the AMES-COND model (Chabrier et al. 2000; Baraffe et al. 2003), for consistency with the other curves. The mass cut-off at $0.5 M_{\text {Jup }}$ is not physical, but due to the mass lower limits in the AMES-COND model. Given the deep sensitivity limits, Beichman et al. (2010) predict that JWST will likely place limits down to Saturn-mass planets and contribute to our understanding of the gap between the inner and outer Vega debris belts.

\section{Conclusions}

We present the results of a deep search for companions around Vega with the Palomar P1640 high-contrast imaging instrument. We combine data from several nights of P1640 IFS data spanning the $J+H$ band. We did not detect any point sources in our data. We present contrast curves and mass limits on Vega from our data (2-15au) and compare these with sensitivity limits with MMT, Spitzer, and predicted JWST limits. JWST data will provide a significant improvement over the previous data beyond $11 \mathrm{au}$, in particular between the warm and cold debris belts. This is complemented by our P1640 sensitivity limits inside $10 \mathrm{au}$ and inside the warm dust belt.

We thank the anonymous referee for their helpful suggestions that improved this paper. We thank the Palomar mountain crew, especially Bruce Baker, Mike Doyle, Carolyn Heffner, John Henning, Greg van Idsinga, Steve Kunsman, Dan McKenna, Jean Mueller, Kajsa Peffer, Paul Nied, Joel Pearman, Kevin Rykoski, Carolyn Heffner, Jamey Eriksen, and Pam Thompson. We thank AAron Veicht for his contributions to the data acquisition during the observations. K.Y.L.S. acknowledges the partial support from the NASA grant NNX15AI86G. G.V. acknowledges the JPL Research \& Technology Program and the NASA XRP grant 399131.02.07.02.66.

\section{ORCID iDs}

Tiffany Meshkat (1) https://orcid.org/0000-0001-6126-2467 Ricky Nilsson (1) https://orcid.org/0000-0002-5408-4954

\footnotetext{
https://pynrc.readthedocs.io
}

Jonathan Aguilar (i https://orcid.org/0000-0003-3184-0873

Gautam Vasisht (1) https://orcid.org/0000-0002-1871-6264

Rebecca Oppenheimer (i) https://orcid.org/0000-00017130-7681

Kate Y. L. Su (1) https://orcid.org/0000-0002-3532-5580

Eric Cady (i) https://orcid.org/0000-0002-8789-2931

Christopher Matthews (1) https://orcid.org/0000-0002-

7782-0272

Jarron Leisenring (i) https://orcid.org/0000-0002-0834-6140

Dimitri Mawet (1) https://orcid.org/0000-0002-8895-4735

\section{References}

Absil, O., di Folco, E., Mérand, A., et al. 2006, A\&A, 452, 237

Amara, A., \& Quanz, S. P. 2012, MNRAS, 427, 948

Baraffe, I., Chabrier, G., Barman, T. S., Allard, F., \& Hauschildt, P. H. 2003, A\&A, 402, 701

Beichman, C. A., Krist, J., Trauger, J. T., et al. 2010, PASP, 122, 162

Cady, E., Baranec, C., Beichman, C., et al. 2013, Proc. SPIE, 8864, 88640K Chabrier, G., Baraffe, I., Allard, F., \& Hauschildt, P. 2000, ApJ, 542, 464

Chiang, E., Kite, E., Kalas, P., Graham, J. R., \& Clampin, M. 2009, ApJ, 693,734

Defrère, D., Absil, O., Augereau, J.-C., et al. 2011, A\&A, 534, A5

Dekany, R., Roberts, J., Burruss, R., et al. 2013, ApJ, 776, 130

Fergus, R., Hogg, D. W., Oppenheimer, R., Brenner, D., \& Pueyo, L. 2014, ApJ, 794, 161

Heinze, A. N., Hinz, P. M., Kenworthy, M., Miller, D., \& Sivanandam, S. 2008, ApJ, 688, 583

Hinkley, S. 2009, PhD thesis, Columbia Univ.

Hinkley, S., Oppenheimer, B., Zimmerman, N., et al. 2011, PASP, 123, 74

Hinkley, S., Oppenheimer, B. R., Soummer, R., et al. 2007, ApJ, 654, 633

Holland, W. S., Matthews, B. C., Kennedy, G. M., et al. 2017, MNRAS, 470,3606

Janson, M., Quanz, S. P., Carson, J. C., et al. 2015, A\&A, 574, A120

Koerner, D. W., Sargent, A. I., \& Ostroff, N. A. 2001, ApJL, 560, L181

Lagrange, A.-M., Bonnefoy, M., Chauvin, G., et al. 2010, Sci, 329, 57

Lagrange, A.-M., Gratadour, D., Chauvin, G., et al. 2009, A\&A, 493, L21

Macintosh, B., Graham, J. R., Barman, T., et al. 2015, Sci, 350, 64

Macintosh, B. A., Becklin, E. E., Kaisler, D., Konopacky, Q., \& Zuckerman, B. 2003, ApJ, 594, 538

Marois, C., Lafrenière, D., Doyon, R., Macintosh, B., \& Nadeau, D. 2006, ApJ, 641, 556

Marois, C., Macintosh, B., Barman, T., et al. 2008, Sci, 322, 1348

Marois, C., Zuckerman, B., Konopacky, Q. M., Macintosh, B., \& Barman, T. 2010, Natur, 468, 1080

Meshkat, T., Kenworthy, M. A., Quanz, S. P., \& Amara, A. 2014, ApJ, 780, 17

Meshkat, T., Mawet, D., Bryan, M. L., et al. 2017, AJ, 154, 245

Metchev, S. A., Hillenbrand, L. A., \& White, R. J. 2003, ApJ, 582, 1102

Morrison, S., \& Malhotra, R. 2015, ApJ, 799, 41

Oppenheimer, B. R., Beichman, C., Brenner, D., et al. 2012, Proc. SPIE, 8447, 844720

Perrin, M. D., Sivaramakrishnan, A., Lajoie, C.-P., et al. 2014, Proc. SPIE, 9143, 91433X

Quillen, A. C. 2006, MNRAS, 372, L14

Rameau, J., Chauvin, G., Lagrange, A.-M., et al. 2013, ApJL, 772, L15

Raymond, S. N., Armitage, P. J., Moro-Martín, A., et al. 2011, A\&A, 530, A62 Raymond, S. N., Armitage, P. J., Moro-Martín, A., et al. 2012, A\&A, 541, A11 Raymond, S. N., \& Bonsor, A. 2014, MNRAS, 442, L18

Sibthorpe, B., Vandenbussche, B., Greaves, J. S., et al. 2010, A\&A, 518, L130

Soummer, R., Pueyo, L., Ferrari, A., et al. 2009, ApJ, 695, 695

Soummer, R., Pueyo, L., \& Larkin, J. 2012, ApJL, 755, L28

Sparks, W. B., \& Ford, H. C. 2002, ApJ, 578, 543

Su, K. Y. L., Rieke, G. H., Malhotra, R., et al. 2013, ApJ, 763, 118

Su, K. Y. L., Rieke, G. H., Misselt, K. A., et al. 2005, ApJ, 628, 487

van Leeuwen, F. 2007, A\&A, 474, 653

Vasisht, G., Cady, E., Zhai, C., Lockhart, T., \& Oppenheimer, B. 2014, Proc. SPIE, 9148, 914822

Wang, J. J., Perrin, M. D., Savransky, D., et al. 2018, JATIS, 4, 018002

Wilner, D. J., Holman, M. J., Kuchner, M. J., \& Ho, P. T. P. 2002, ApJL, 569, L115

Wyatt, M. C. 2008, ARA\&A, 46, 339

Yoon, J., Peterson, D. M., Kurucz, R. L., \& Zagarello, R. J. 2010, ApJ, 708, 71 Zhai, C., Vasisht, G., Shao, M., et al. 2012, Proc. SPIE, 8447, 84476W

Zimmerman, N., Brenner, D., Oppenheimer, B., et al. 2011, PASP, 123, 746 